\title{
Characterisation methods for the hyperspectral sensor HySpex at DLR's calibration home base
}

\author{
Andreas Baumgartner, Peter Gege, Claas Köhler, Karim Lenhard, Thomas Schwarzmaier, \\ Deutsches Zentrum für Luft- und Raumfahrt (DLR), Earth Observation Center, Remote \\ Sensing Technology Institute, Oberpfaffenhofen, 82234 Wessling, Germany
}

\begin{abstract}
The German Aerospace Center's (DLR) Remote Sensing Technology Institute (IMF) operates a laboratory for the characterisation of imaging spectrometers. Originally designed as Calibration Home Base (CHB) for the imaging spectrometer APEX, the laboratory can be used to characterise nearly every airborne hyperspectral system. Characterisation methods will be demonstrated exemplarily with HySpex, an airborne imaging spectrometer system from Norsk Elektro Optikks A/S (NEO). Consisting of two separate devices (VNIR-1600 and SWIR-320me) the setup covers the spectral range from $400 \mathrm{~nm}$ to $2500 \mathrm{~nm}$. Both airborne sensors have been characterised at NEO. This includes measurement of spectral and spatial resolution and misregistration, polarisation sensitivity, signal to noise ratios and the radiometric response. The same parameters have been examined at the CHB and were used to validate the NEO measurements. Additionally, the line spread functions (LSF) in across and along track direction and the spectral response functions (SRF) for certain detector pixels were measured. The high degree of lab automation allows the determination of the SRFs and LSFs for a large amount of sampling points. Despite this, the measurement of these functions for every detector element would be too time-consuming as typical detectors have $10^{5}$ elements. But with enough sampling points it is possible to interpolate the attributes of the remaining pixels. The knowledge of these properties for every detector element allows the quantification of spectral and spatial misregistration (smile and keystone) and a better calibration of airborne data. Further laboratory measurements are used to validate the models for the spectral and spatial properties of the imaging spectrometers. Compared to the future German spaceborne hyperspectral Imager EnMAP, the HySpex sensors have the same or higher spectral and spatial resolution. Therefore, airborne data will be used to prepare for and validate the spaceborne system's data.
\end{abstract}

Keywords: Hyperspectral, Imaging Spectrometer, Smile, Keystone, Calibration, Radiometry, LSF, SRF

\section{INTRODUCTION}

DLR's Remote Sensing Technology Institute (IMF) recently acquired a sensor suite for airborne hyperspectral imaging, a NEO (Norsk Elektro Optikk A/S) ${ }^{1}$ HySpex VNIR-1600 and a NEO HySpex SWIR-320m-e. ${ }^{2}$ The combined wavelength range covers the spectrum between $410 \mathrm{~nm}$ and $2500 \mathrm{~nm}$. Since this sensor suite will be used as a simulator for the hyperspectral mission EnMAP, ${ }^{3}$ its properties need to be characterized in detail. The characterization is performed at DLR's calibration laboratory for imaging spectrometers ${ }^{4}$ in Oberpfaffenhofen, which is also the calibration home base (CHB) for APEX. ${ }^{5}$

The main properties of the imaging spectrometers are listed in table 1. For typical in-flight measurements, both sensors are equipped with field of view (FOV) expander lenses that approximately double the FOVs in order to reduce the number of flight lines required to cover a target area.

In the following chapters the used procedures for the geometric, spectral and radiometric calibration are described. Since the methods are the same for every sensor, they are only shown for the VNIR sensor with field of view expander.

Further author information:

Andreas Baumgartner: E-mail: andreas.baumgartner@dlr.de, Telephone: +49 (0) 8153281402 


\begin{tabular}{l|c|c} 
& VNIR-1600 & SWIR-320m-e \\
\hline Spectral range & $410-1000 \mathrm{~nm}$ & $1000-2500 \mathrm{~nm}$ \\
Sampling interval & $3.7 \mathrm{~nm}$ & $6 \mathrm{~nm}$ \\
FOV & $17^{\circ}$ & $14^{\circ}$ \\
Pixel / line & 1600 & 320 \\
Channels & 160 & 256 \\
Min. GSD & $0.3 \mathrm{~m}$ & $0.6 \mathrm{~m}$ \\
Radiometric resolution & $12 \mathrm{bit}$ & $14 \mathrm{bit}$
\end{tabular}

Table 1. Properties of the HySpex imaging spectrometers without field of view expander.

\section{GEOMETRIC CALIBRATION}

Geometric properties of an imaging spectrometer are described by the geometric Line Spread Functions (LSF). A LSF is defined as the dependency of the sensitivity of a detector element on the viewing angle. LSFs are distinguished between along track (in flight direction) and across track (perpendicular to the flight direction) LSFs. Thus, along track LSFs describe what a detector element "sees" in flight direction and the across track LSFs perpendicular to it. LSFs are usually summarized by their center angles and their Full Width at Half Maximum (FWHM). The FWHM is often used as definition for the Instantaneous Field Of View (IFOV). In an ideal sensor, all channels of a spatial pixel would have the same viewing geometry. In real sensors, both the center angles and the FWHMs are channel dependent. The channel dependency of the viewing angle is called keystone effect.

\subsection{Geometric Measurement Setup}

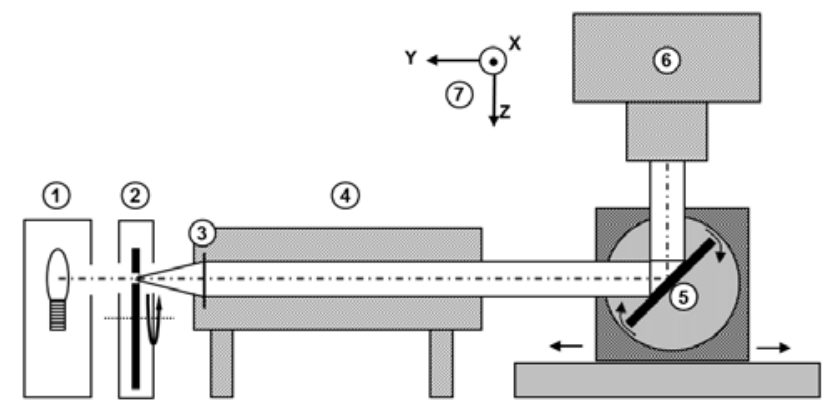

Figure 1. Set-up for geometric measurements. $1=$ Lamp, $2=$ Slit in turnable wheel, $3=$ Parabolic mirror, $4=$ Collimator, $5=$ Folding mirror, $6=$ Sensor, 7 . Laboratory coordinate system. ${ }^{4}$

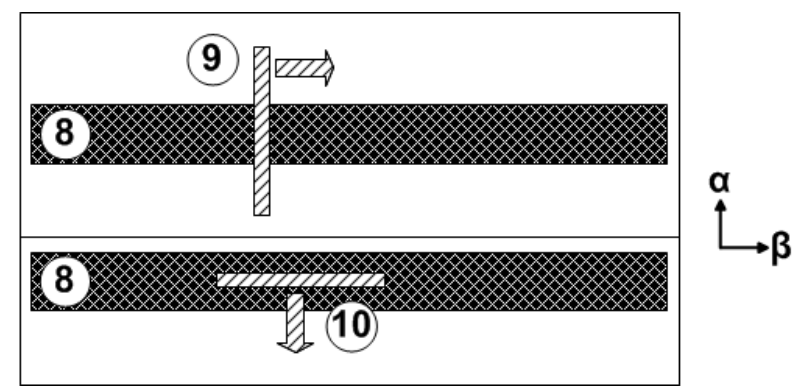

Figure 2. Schematic drawing of the across (top) and along (bottom) track LSF measurement principle. $8=$ Sensor FOV in along ( $\alpha$, rotation around y axis) and $\operatorname{across}(\beta$, rotation around $\mathrm{x}$ axis) track direction, $9=$ Across, $10=$ Along track FOV scanning with a slit.

The setup for measuring geometric sensor properties is shown in Fig. 1. A lamp (1) illuminates a slit (width: $50 \mu \mathrm{m}$ ) on a turnable wheel (2). The slit is located at the focal plane of a collimator (4). The parabolic mirror of the collimator (3) forms a beam of highly parallel light. This beam is reflected by a folding mirror (5) (accuracy: $\pm 0.0053 \mathrm{mrad}$, repeatability: $\pm 0.0010 \mathrm{mrad}$ ) into the entrance aperture of the sensor. The diameter of the collimated beam is larger than the sensor aperture. The sensor entrance slit is adjusted parallel to the y axis of the laboratory coordinate system (7).

Figure 2 shows the measurement principle for the sensor's across ( $\beta$, rotation around $\mathrm{x}$ axis) and along $(\alpha$, rotation around y axis) track LSFs (8). The across track LSFs are measured by imaging a slit perpendicular to the sensor aperture. By moving the folding mirror along the $\mathrm{y}$ axis and rotating it around the $\mathrm{z}$ axis the $\beta$ angle of the slit is changed (9). At 18 equally over the across track FOV distributed points, the across track LSFs of a spatial pixel have been measured. The scan range at each point was $3.5 \mathrm{mrad}$ with a step width of $0.07 \mathrm{mrad}$ (51 sample points). 
The along track LSFs are measured by imaging a slit parallel to the sensor aperture and moving the slit in the object plane of the collimator. Moving the slit is done by rotating the slit wheel. The resulting displacement of the slit is assumed to be linear for the small rotation range. This leads to a change of the incidence angle $\alpha$ of the beam (10). Translation of the folding mirror allows selecting different spatial pixels (causes the slit image (10) to rotate around $\beta$ ). This was done at 18 different across track FOV positions. At each position, 21 along track angles ranging from $-1.51 \mathrm{mrad}$ to $+1.51 \mathrm{mrad}$ were selected.

\subsection{Derivation of Geometric Parameters}

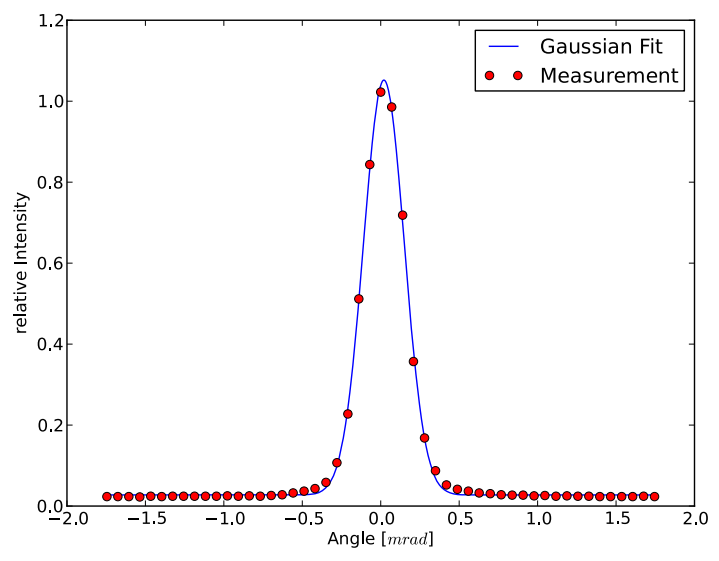

Figure 3. Across track LSF of Pixel 803, Channel 100.

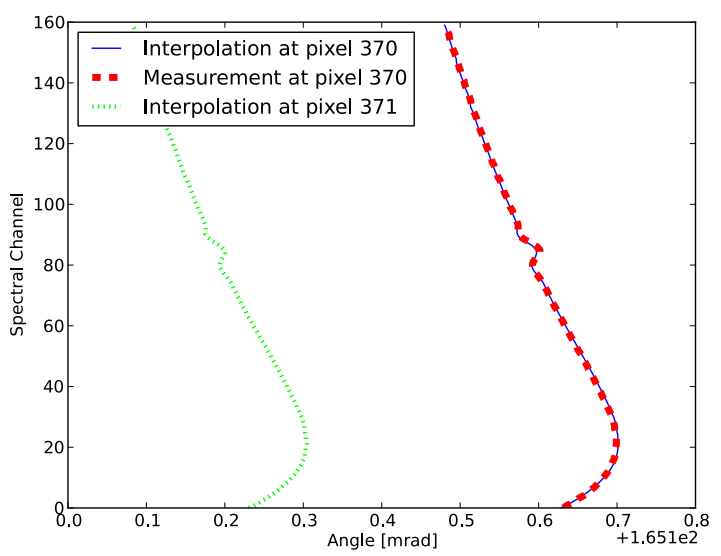

Figure 5. Interpolation of the across track center angles at pixel 370 compared with measurement at the same pixel.

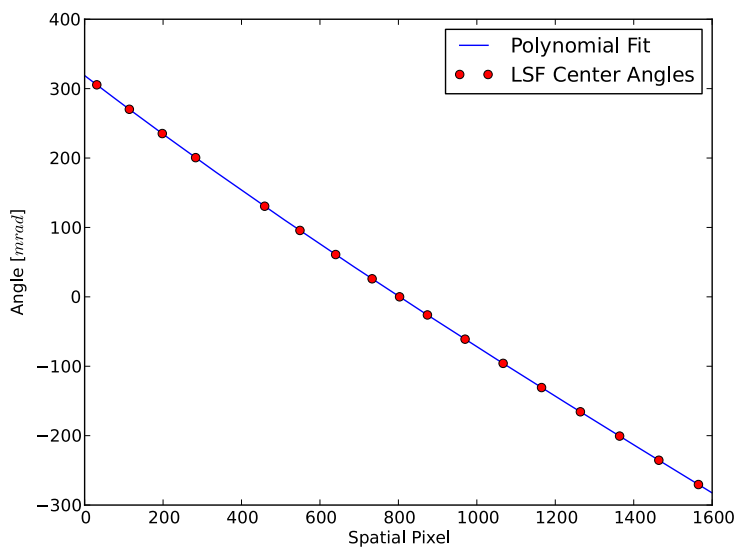

Figure 4. Center angles of the LSFs of Channel 100 with 4th order polynomial fit.

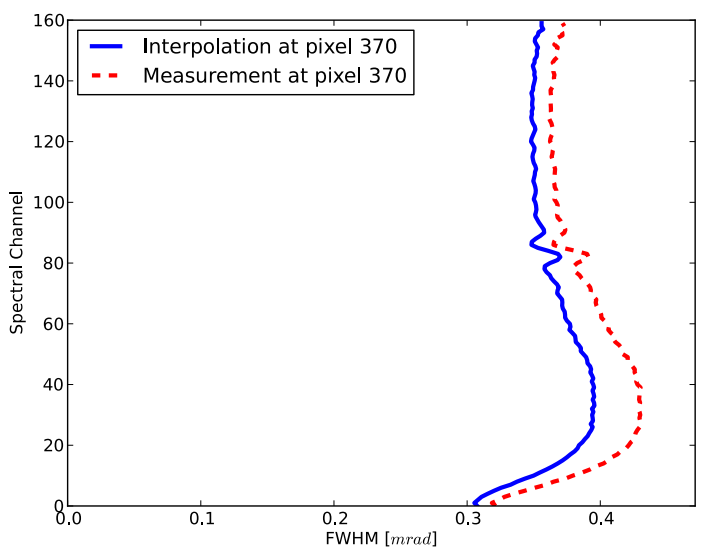

Figure 6. Interpolation of spatial across track FWHMs at pixel 370 compared with measurement at the same pixel.

For each of the 18 across track LSF measurements mentioned in the previous subsection, a spatial pixel was selected, consisting of 160 spectral channels. For every channel the across track LSF has been determined with a Gaussian fit. As an example, Fig. 3 shows the LSF of channel 100 of the spatial pixel 803. The center angles of the LSFs of one channel were used to interpolate the center angles of the remaining pixels (see Fig. 4). As interpolation function a polynomial of 4 th order was used. The interpolated center angles of pixel 370 are plotted in Fig. 5. To outline the order of magnitude of the keystone the central angles of the neighbor pixel 371 are also shown. For these spatial pixels, the keystone is $56 \%$ of a pixel. Verification of the interpolation was done with an additional measurement at pixel 370, which was not taken into account for the interpolation. The difference 
between fit and measurement is below $1 \%$ in respect to the pixel distance. The next sample points are at pixel 283 and pixel 459.

Just as it was done for the center angles the interpolation was done with a polynomial of 4th order for the FHWMs. The absolute difference between interpolation and measurement at pixel 370 is below $6 \%$ (see Fig. 6).

Since the evaluation method for the along track LSFs is nearly the same as for the across track LSFs, it is not described in detail. Like for the along track LSF, a Gaussian function was fitted on the sample points. The way of interpolation is similar, but a 2nd order polynomial turned out to be sufficient here.

After the interpolation process four arrays were generated, which describe the geometric sensor characteristics. Two contain the along and across track center angles and the other the corresponding FWHMs. Figure 14 shows the across track center angles referenced to the angles of channel 80 and Fig. 15 shows the respective FWHMs. The viewing angle and FWHM in along track direction are shown in Fig. 16 and 17. Between channel 75 and channel 90 are noticeable deformations. These deformations are caused by a spectral order blocking filter* which is mounted on top of higher number channels (see also Sec. 3.2).

\section{SPECTRAL CALIBRATION}

Spectral characteristics of a detector element are described by the Spectral Response Function (SRF). SRFs are the dependency of sensor pixels sensitivity on the wavelength of the incident light. Similar to LSFs, the main parameters are the center wavelength (instead of center angle) and the FWHM describing the spectral resolution. Like the keystone effect for center angles, for most sensors there is a change of the center wavelength of one channel with the spatial pixel. This effect is called spectral smile.

\subsection{Spectral Measurement Setup}

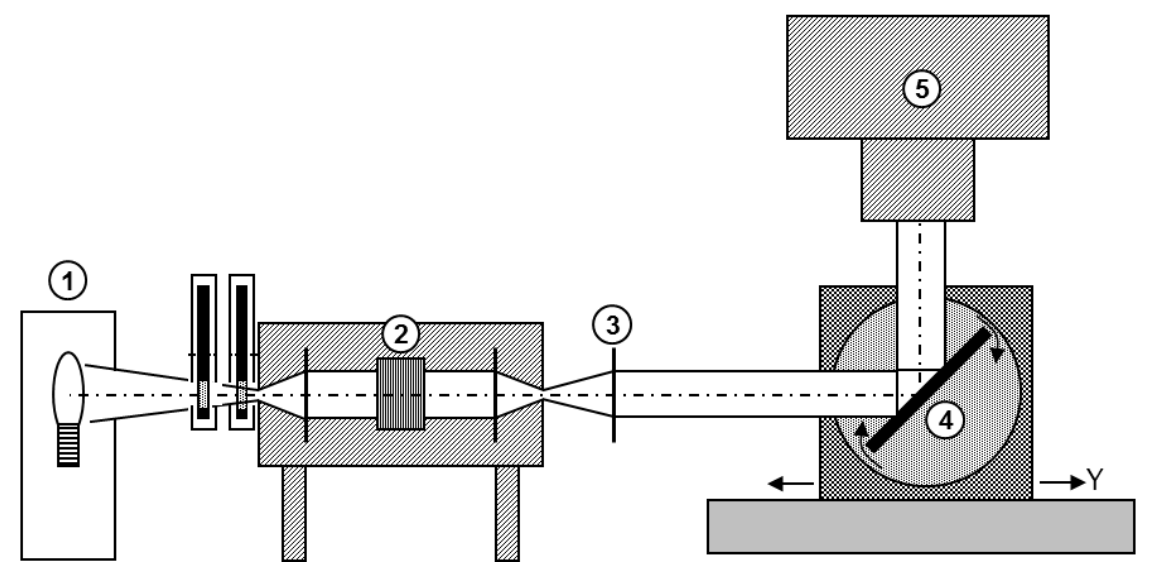

Figure 7. Set-up for spectral measurements. $1=$ Lamp, $2=$ Monochromator, $3=$ Parabolic mirror, $4=$ Folding mirror, 5 = Sensor. ${ }^{4}$

The spectral calibration setup is shown in Fig. 7. In this setup light (1) is dispersed by a monochromator(2). The nearly monochromatic light from the monochromator slit is collimated (3) and guided over a folding mirror (4) to the sensor aperture (5). To guarantee that the entrance aperture and the along track FOV are completely illuminated, the beam diameter is larger than the sensor aperture and the beam divergence is larger then the sensor's along track FOV. The beam angle with respect to the sensor's optical axis was changed by moving and rotating the folding mirror. Different beam angles result in the illumination of different spatial pixels (see Sec. 2).

The used monochromator has an absolute accuracy of $\pm 0.15 \mathrm{~nm}$ and the spectral bandwidth was set to 0.65 $\mathrm{nm}$. For the described measurements, the wavelength has been tuned from $410 \mathrm{~nm}$ to $1010 \mathrm{~nm}$ in $1 \mathrm{~nm}$ steps.

${ }^{*}$ Information from NEO 
This has been done for nine spatial pixels, equally distributed over the sensor's across track FOV. Additionally measurements have been performed from $750 \mathrm{~nm}$ to $770 \mathrm{~nm}$ at 35 spatial pixels was performed, to study in detail the sensor behavior around the oxygen absorption line at $762 \mathrm{~nm}$.

\subsection{Derivation of Spectral Parameters}

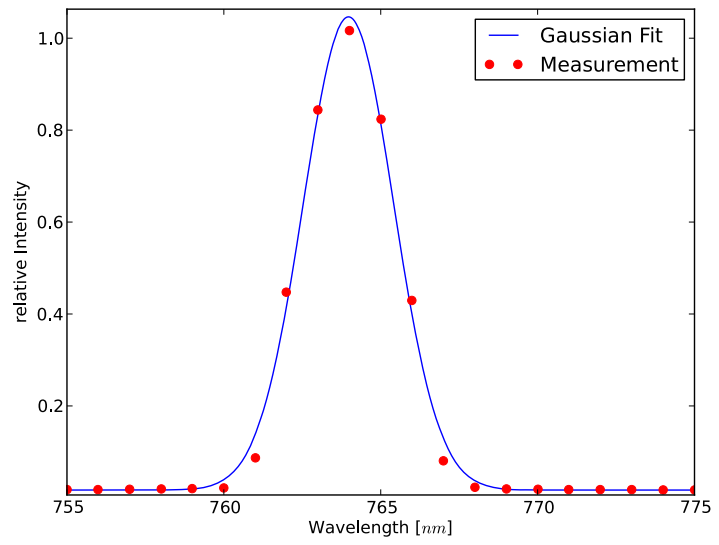

Figure 8. SRF of pixel 805, channel 96.

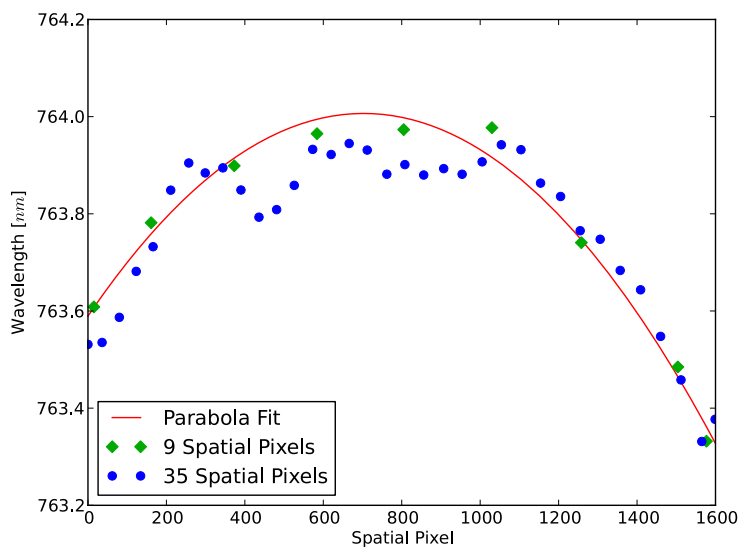

Figure 10. Center wavelengths of channel 96. Nine, equally distributed measurement point with the associated parabola fit and 35 points for validation of the fit.

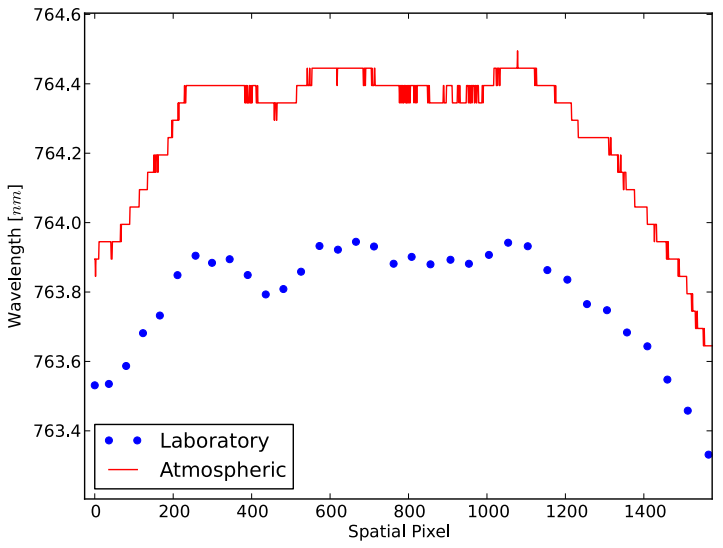

Figure 9. Center wavelengths of channel 96.

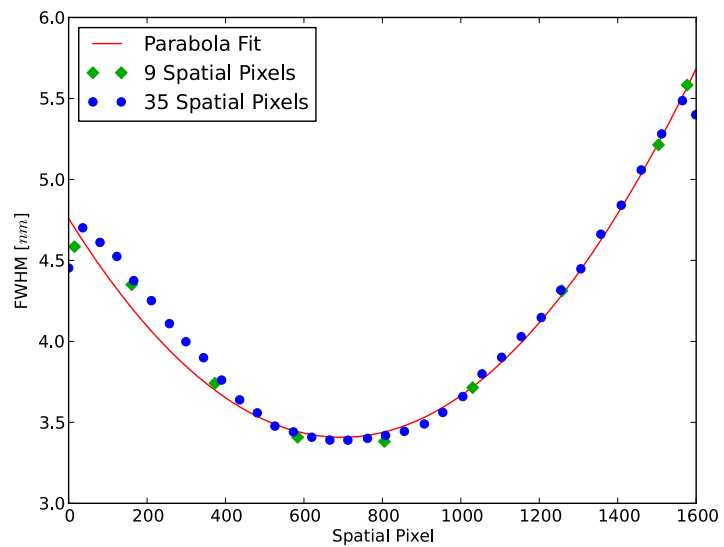

Figure 11. Spectral FHWM's of channel 96. Nine, equally distributed measurement point with the associated parabola fit and 35 points for validation of the fit.

For each spectral channel of the investigated spatial pixels the center wavelength and FWHM of the SRF have been determined. This has been done by fitting a Gaussian function on the measured data (see Fig. 8). Then a parabola (2nd order polynomial) has been fitted on the center wavelengths for every channel. These polynomials have been used to interpolate the properties of the remaining detector elements. An example of a polynomial fit on nine center wavelengths of channel 96 is shown in Fig. 10. Additionally, the center wavelengths of 35 spatial pixels for the same channel in are shown. It appears that the shape of the smile is not exactly parabolic. However, as the difference to the interpolation parabola is in the order of the uncertainty of the monochromator, a parabola is used to describe the spectral characteristics of the remaining channels.

To verify the measured shape of the spectral smile, the center wavelengths of channel 96 were derived with ATCOR $^{6}$ from flight data (see Fig. 9). The shape of the smile measured in the laboratory is comparable to the 
data derived with ATCOR. However, there is a systematic shift between both graphs of approximately $0.5 \mathrm{~nm}$. The reason for this shift is not yet clear and part of further investigations.

In the same way as it was done for the center wavelengths, the FWHMs have been interpolated with a parabola and validated with 35 measurement points (see Fig. 11). As the figure shows, a parabola is a good approximation for the distribution of the FWHMs over the detector.

By applying the described procedures, two $1600 \times 160$ spectral calibration arrays are derived. One with the center wavelengths and the other with the spectral resolution for each detector element. Figure 18 and Fig. 19 show the calculated smile and FHWM distribution on the detector. Between channel 75 (687.9 nm @ pixel 800) and 90 (742.2 nm @ pixel 800) higher smile effects and larger FWHMs can be observed. This is caused by a spectral order blocking filter which is mounted over the higher number channel. Its edge leads to a deformation of the SRFs in this area, which can not be well described by the Gaussian model chosen for the SRFs. This effect is also visible in the geometric calibration (see Sec. 2.2).

\section{RADIOMETRIC MEASUREMENTS}

\subsection{Radiometric calibration}

The CHB's radiance standard (RASTA) ${ }^{7}$ consists of a combination of a spectral panel and a 1000 W-FEL lamp. Additionally, five filter radiometers monitor the emitted spectral radiance of the standard to make sure that the calibration performed by the German national metrology institute $(\mathrm{PTB})^{8}$ is still valid. For the actual calibration of the HySpex instruments, an integrating sphere was used as light source. This integrating sphere is linked to RASTA via a spectrometer (in this case: SVC HR-1024) measurements. The radiometric calibration coefficients for the nadir pixels of both devices is shown in Fig. 12 for the configuration without FOV expander.

\subsection{Noise equivalent spectral radiance}

The noise equivalent spectral radiance (NESR) was determined for both devices by determining the dark current's standard deviation for an integration time of $1 \mathrm{~ms}$ and performing a radiometric calibration with the coefficients shown in Fig. 12. An integration time of $1 \mathrm{~ms}$ is the lowest set able in-flight value, typical in-flight integration times are more than ten times higher. The result can be seen in Fig. 13.

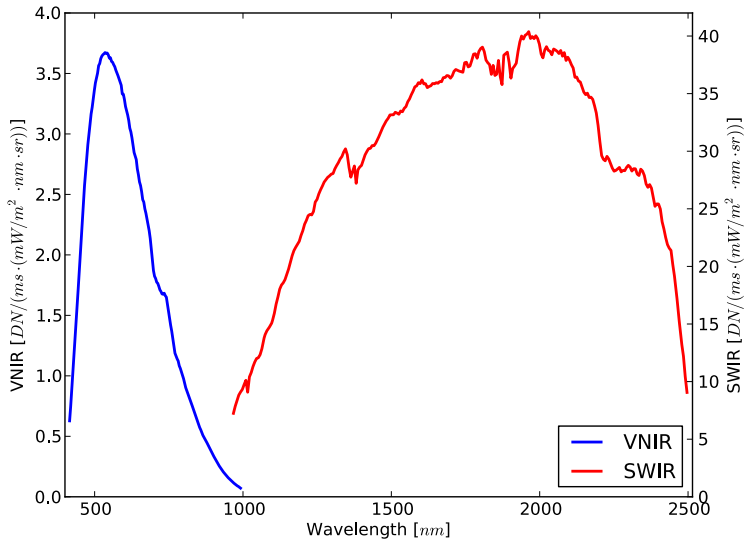

Figure 12. Radiometric calibration coefficients of both sensors without FOV expander.

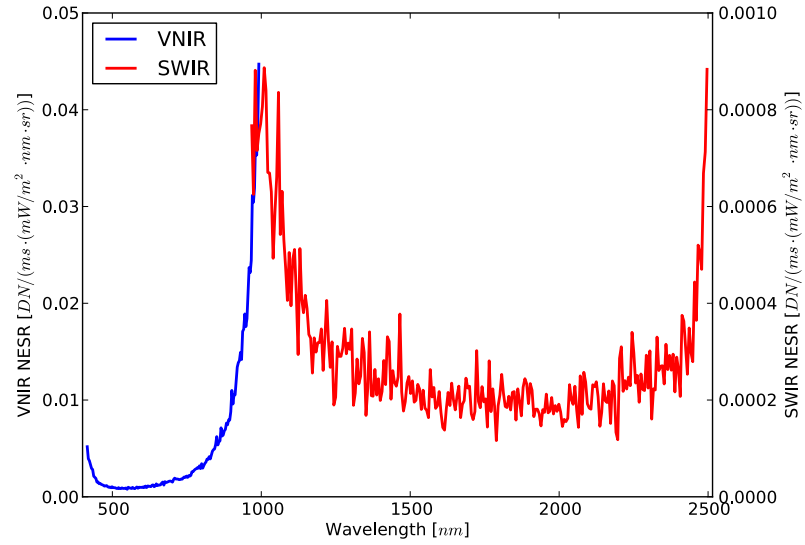

Figure 13. Noise equivalent spectral radiance for the nadir pixels of the VNIR and SWIR sensors without FOV expander. 


\section{CONCLUSION}

The calibration methods outlined above show the potential of a highly automated laboratory. More than 8000 single measurements were done for the spatial and spectral calibration mentioned in this paper. Doing this manually in acceptable time is impossible. Nevertheless measuring the spatial and spectral properties of every detector element is still not reachable in acceptable time. So the characteristics of all pixels have to be interpolated out of decent sample points. A way to do such interpolations and according verifications were shown. Although the most important characteristics of the sensor were evaluated, some measurements, like stray light and polarisation characterisation, are still missing. Also an accurate description of measurement uncertainties is an important task for the future.

\section{ACKNOWLEDGMENTS}

The authors want to acknowledge Rudolf Richter (DLR) for calculating the center wavelengths of channel 96 of the VNIR sensor with ATCOR and Trond Løke (NEO) for his helpful and quick responses on our numerous questions.

\section{REFERENCES}

[1] Optikk, N. E., "Website of norsk elektro optikk." http://www.neo.no/ (Jan. 2012).

[2] Optikk, N. E., "Website of norsk elektro optikk for hyspex sensors." http://www.hyspex.no/ (Jan. 2012).

[3] Kaufmann, H., Segl, K., Chabrillat, S., Hofer, S., Stuffler, T., and Mueller, A., "Enmap a hyperspectral sensor for environmental mapping and analysis," IGARSS Proceedings (2006).

[4] Gege, P., Fries, J., Haschberger, P., Schoetz, P., Schwarzer, H., Strobl, P., Suhr, B., Ulbrich, G., and Vreeling, W., "Calibration facility for airborne imaging spectrometers," ISPRS Journal of Photogrammetry \&3 Remote Sensing 64, 387-397 (2009).

[5] Itten, K., Dell'Endice, F., Hueni, A., Kneubhler, M., Schlaepfer, D., Odermatt, D., Seidel, F., Huber, S., Schopfer, J., Kellenberger, T., Buehler, Y., D'Odorico, P., Nieke, J., Alberti, E., and Meuleman, K., "Apex - the hyperspectral esa airborne prism experiment," Sensors 8, 6235-6259 (2008).

[6] Richter, R., Schlaepfer, D., and Mueller, A., "Operational atmospheric correction for imaging spectrometers accounting for the smile effect," IEEE Transactions on Geoscience and Remote Sensing 49, 1172-1780 (May 2011).

[7] Schwarzmaier, T., Baumgartner, A., Gege, P., Koehler, C., and Lenhard, K., "The radiance standard rasta of dlr's calibration facility for airborne imaging spectrometers," in [SPIE Remote Sensing, Edinburgh, United Kingdom], (September 2012).

[8] Taubert, D., Hollandt, J., Sperfeld, P., Hpe, A., Pape, S., Gege, P., Schwarzmaier, T., Lenhard, K., and Baumgartner, A., "Providing radiometric traceability for the calibration home base of dlr by ptb," in [International Radiation Symposium, Berlin, Germany], (August 2012). 


\section{APPENDIX}

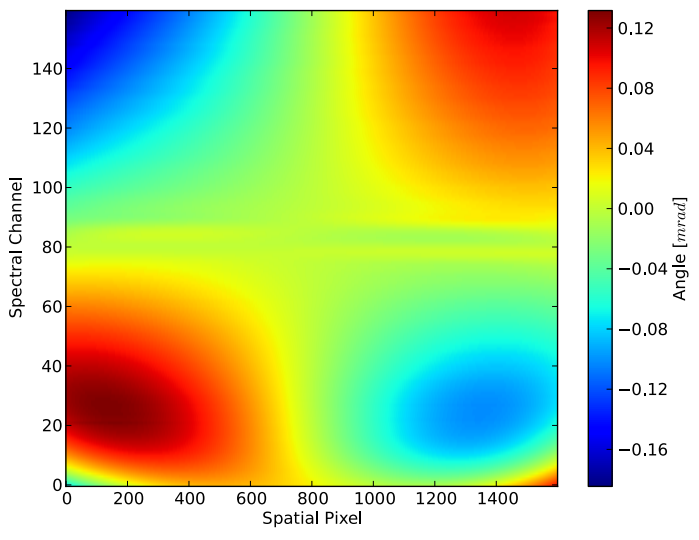

Figure 14. Geometric across track keystone: $\beta$ $\beta_{\text {Channel80. }}$

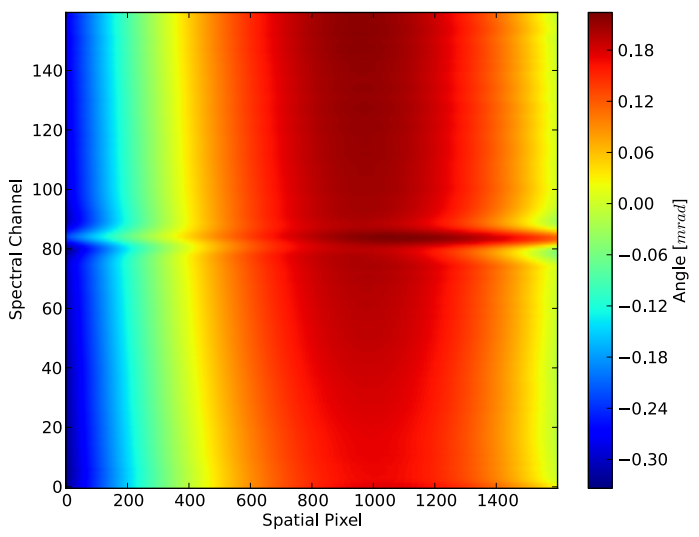

Figure 16. Along track viewing angle $\alpha$.

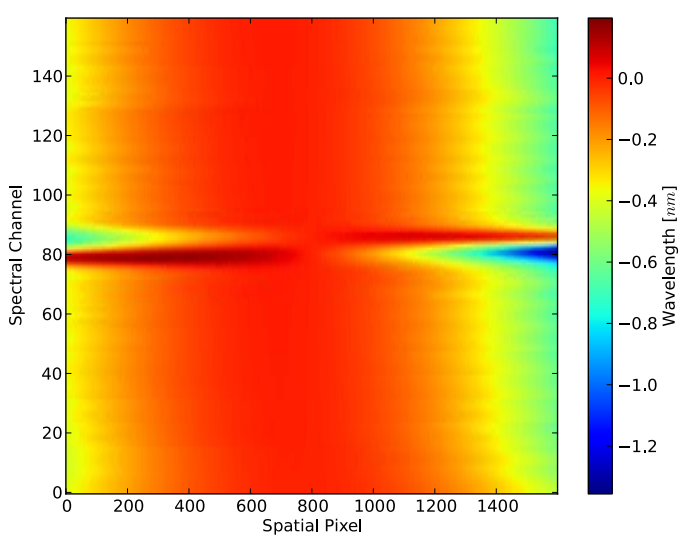

Figure 18. Spectral smile: $\lambda$ - $\lambda_{\text {Pixel800 }}$.

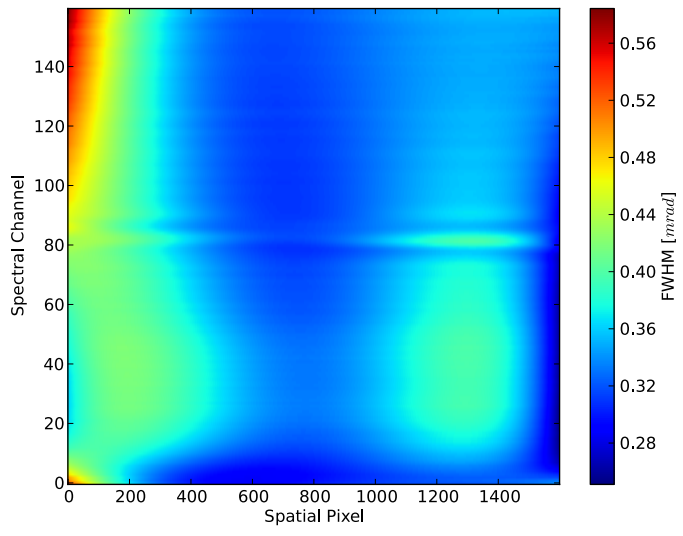

Figure 15. Geometric across track resolution (FWHM).

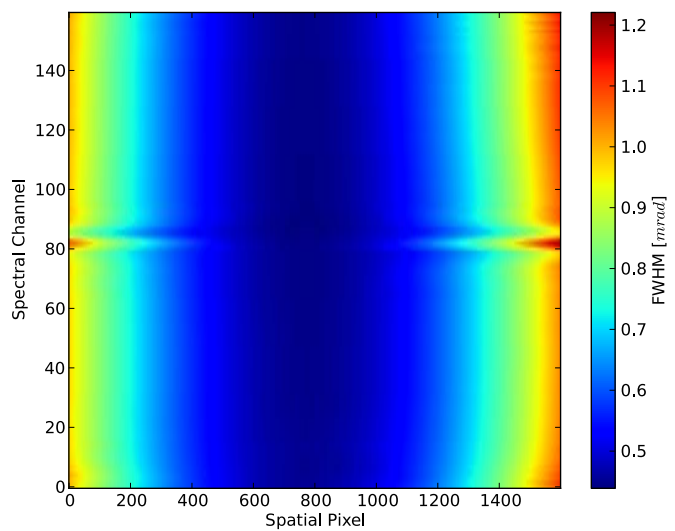

Figure 17. Geometric along track resolution (FWHM).

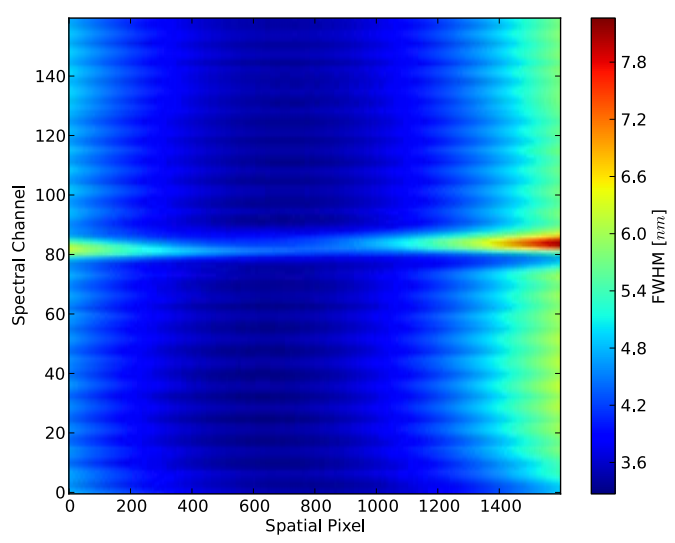

Figure 19. Spectral resolution (FWHM). 